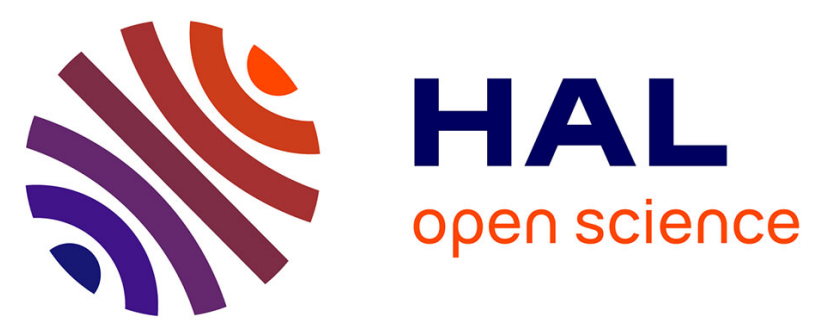

\title{
Desorption Electrospray Ionization (DESI) of Digital Polymers: Direct Tandem Mass Spectrometry Decoding and Imaging from Materials Surfaces
}

Jean-arthur Amalian, Tathagata Mondal, Evgeniia Konishcheva, Gianni Cavallo, Benoît Eric Petit, Jean-François Lutz, Laurence Charles

\section{To cite this version:}

Jean-arthur Amalian, Tathagata Mondal, Evgeniia Konishcheva, Gianni Cavallo, Benoît Eric Petit, et al.. Desorption Electrospray Ionization (DESI) of Digital Polymers: Direct Tandem Mass Spectrometry Decoding and Imaging from Materials Surfaces. Advanced Materials Technologies, 2021, 6 (4), pp.2001088. 10.1002/admt.202001088 . hal-03201719

\section{HAL Id: hal-03201719 \\ https://hal.science/hal-03201719}

Submitted on 30 Nov 2021

HAL is a multi-disciplinary open access archive for the deposit and dissemination of scientific research documents, whether they are published or not. The documents may come from teaching and research institutions in France or abroad, or from public or private research centers.
L'archive ouverte pluridisciplinaire HAL, est destinée au dépôt et à la diffusion de documents scientifiques de niveau recherche, publiés ou non, émanant des établissements d'enseignement et de recherche français ou étrangers, des laboratoires publics ou privés. 


\title{
Desorption electrospray ionization (DESI) of
}

\section{digital polymers: direct tandem mass spectrometry decoding and imaging from materials surfaces}

Jean-Arthur Amalian, Tathagata Mondal, Evgeniia Konishcheva, Gianni Cavallo, Benoît Eric Petit, Jean-François Lutz, * and Laurence Charles*

\author{
Dr. J.-A. Amalian, Prof. Dr. L. Charles \\ Aix Marseille Université, CNRS, UMR 7273 \\ Institute of Radical Chemistry \\ 13397 Marseille Cedex 20, France \\ E-mail: laurence.charles@univ-amu.fr
}

Dr. T. Mondal, Dr. E. Konishcheva, Dr. G. Cavallo, Dr. B.E. Petit, Dr. J.-F. Lutz Université de Strasbourg, CNRS, Institut Charles Sadron UPR 22 Precision Macromolecular Chemistry 23, rue du Loess, 67034 Strasbourg Cedex 2, France E-mail: jflutz@unistra.fr

Published in Advanced Materials Technologies, 6, 2001088 (2021) https://onlinelibrary.wiley.com/doi/abs/10.1002/admt.202001088 
Abstract. Sequence-defined synthetic polymers have recently emerged as an attractive medium to store information at the molecular level, where co-monomers of the chains are defined as letters of an alphabet. The main read-out methodology employed to retrieve such molecularly encoded information is tandem mass spectrometry (MS/MS), but a major current limitation remains the low storage capacity of readable chains. Ordering short oligomers at discrete locations onto surfaces to compose long messages is an attractive alternative to the difficult synthesis of long coded polymers. Yet, such surface storage requires a reliable sampling technique to be coupled on-line with MS/MS. Because it combines fast surface extraction with efficient analyte ionization in ambient conditions, desorption electrospray ionization (DESI) is shown here to be perfectly suited to envisage bidimensional data storage. The present study demonstrates performances of DESI-MS/MS at mapping oligomers used to write letters of a word, extracting digital labels from materials tagged for anticounterfeiting purposes, and imaging text written with coded polymeric inks. 
Over the last ten years, a variety of synthetic polymers has been developed with a defined sequence of monomers, defined as letters of an alphabet, to store information at the molecular level. $^{[1-9]}$ Since decoding this information is typically a sequencing task, tandem mass spectrometry (MS/MS) has early been acknowledged as the most efficient method to read such molecularly encoded messages. This technique is renowned for its speed, selectivity and sensitivity, and has become a routine technique in many laboratories, all key advantages contributing to widespread applications of digital polymers. Yet, a main current issue remains the storage capacity of MS/MS-readable chains. Iterative synthesis is used to produce sequencedefined polymers by attaching co-monomers one by one in a given order, and the efficiency of these protocols is limited to chains containing up to $100-150$ repeating units. ${ }^{[9,10]}$ Still, such chain-lengths are far above the upper limit of 28 repeating units ever demonstrated for de novo sequencing of digitally-encoded polymers by MS/MS. ${ }^{[11,12]}$ Indeed, in order to assess monomer connectivity along the whole chain, the same dissociation reactions have to proceed at all repeating units: the number of fragments rapidly increases with the degree of polymerization (DP), leading to signal dilution which hampers detectability of sequencing fragments. To address this issue, the chemical structure of monomers can be optimized to decrease their MS/MS reactivity and hence lower the number of dissociation pathways per repeating unit. ${ }^{[13 \text {, }}$ ${ }^{14]}$ Using this strategy, the longest chain read so far by MS/MS alone is a 77-mer. ${ }^{[4]}$ Increasing the number of encoded bits per monomer is another efficient strategy to enhance the storage capacity of a single chain. ${ }^{[5,15]}$ Increasingly complex alphabets have recently been reported, ${ }^{[6,}$ 7,11,16-19] but their use was mostly demonstrated for short oligomers with DP below 20. However, combining monomer design and storage density control (3 bits/monomer) was recently reported to yield high-capacity MS/MS-readable digital polymers with up to 144 bits/chain. ${ }^{[20]}$

An alternative to the production of long chains to store large amounts of information is to combine short, more readily available, oligomers to write messages. For example, our group used a set of six uniform oligo(alkoxyamine amide)s ${ }^{[3]}$ of different DPs $(2,4,5,6,7$, and 8 , 
respectively) to compose a 4-bytes extended ASCII-coded message. ${ }^{[21]}$ This mixture leads to polydisperse patterns in MS, from which each ion can be individually sequenced by MS/MS. Summing sequence information of all components allows the full binary message to be reconstructed, providing that reading rules are defined for both dimensions. In this previous work, simple rules regarding reading direction were i) by increasing molar mass in the first dimension (MS) and ii) from $\alpha$ to $\omega$ terminus in the second dimension (MS/MS). The main limitation of such a 2D coding approach is the need for all components of the message to have different masses so that they can be distinguished in MS, which requires a quite large library of oligomers to envisage a great variety of messages.

An attractive solution to tackle this issue is multi-dimensional storage, ${ }^{[22]}$ with digital polymers ordered at discrete locations onto surfaces $(2 \mathrm{D})^{[23]}$ or in layers (3D). ${ }^{[24]}$ While there is no method currently available to read 3D patterns by MS/MS, ionization techniques capable of surface sampling have been used to decipher molecularly-encoded information. However, so far, these techniques were not coupled to MS/MS sequencing of digital oligomers but implemented in the MS mode to read messages composed with small molecules. For example, Whitesides et coll. used self-assembled monolayer laser desorption/ionization (SAMDI) ${ }^{[25]}$ to reveal oligopeptides immobilized on different spots of self-assembled monolayer plates: the presence or absence of any given component in the mass analyzed mixtures prepared from a library of 32 oligopeptides was used as the way to code for $\mathbf{1}$ or $\mathbf{0}$, respectively. ${ }^{[26]}$ Employing the same (presence/absence) coding strategy, the Rosenstein's group employed matrix-assisted laser/desorption ionization (MALDI) ${ }^{[27,28]}$ to analyze individual wells of a sample plate where mixtures were prepared from either sets of metabolites ${ }^{[29]}$ or large libraries of Ugi products. ${ }^{[30]}$ Using supervised learning algorithms to identify library components detected in each mass spectrum, reading of digital pictures of up to about $880 \mathrm{kbits}$ was reported. ${ }^{[30]}$

An alternative technique with surface sampling capabilities is desorption electrospray ionization (DESI), ${ }^{[31]}$ an ambient ionization method which makes use of an electrosprayed 
solvent to examine sample surfaces at atmospheric pressure. High velocity charged droplets produced by the ESI probe are directed to the surface where they form a thin film enabling analyte dissolution; further impact of primary charged droplets at the sample surface creates secondary droplets carrying analyte molecules that are ultimately emitted as intact gas phase ions and sampled in the mass spectrometer via a transfer line. ${ }^{[32]}$ As an ambient method, DESI analysis is performed on samples in their native state, with no need for any preparation step such as matrix adjunction as required in MALDI. DESI has been applied to a wide variety of analytes, including synthetic polymers, ${ }^{[33-36]}$ and enables routine analyses on different substrates within a few seconds. Moreover, combining DESI with an automated surface stage capable of translational motion offers capability for $2 \mathrm{D}$ molecular imaging of surfaces. ${ }^{[37,38]}$ These features make DESI an attractive method for MS/MS readout of digital polymers organized on surfaces. Herein, proof-of-principle experiments include three case studies using different families of coded oligomers (Table 1): mapping discrete oligomers used as letters of a word, direct decoding of an anticounterfeiting tag hidden in the gum of a sealed envelope and imaging pictures drawn with digital inks.

Table 1. Structural features of sequence-defined oligomers and experimental DESI conditions.

\begin{tabular}{|c|c|c|c|c|}
\hline \multicolumn{2}{|c|}{ Coded oligomers } & & \multicolumn{2}{|l|}{ DESI solution } \\
\hline Name & Mass [Da] & Binary sequence & Composition & Flow rate \\
\hline \multicolumn{2}{|c|}{$\frac{\text { Oligourethanes }}{\text { (case study \#1) }}$} & & & ; $=\mathrm{CH}_{3} \rightarrow 1$ \\
\hline P1 & 634.4 & 0111 & \multirow{5}{*}{$\mathrm{MeOH}+3 \mathrm{mM} \mathrm{AcNH}_{4}$} & \\
\hline $\mathrm{P} 2$ & 620.4 & 0011 & & \\
\hline P3 & 606.4 & 0100 & & $2 \mu \mathrm{L} \min ^{-1}$ \\
\hline P4 & 620.4 & 1001 & & \\
\hline P5 & 606.4 & 1000 & & \\
\hline \multicolumn{3}{|c|}{$\frac{\text { Oligo(N-substituted urethane)s }}{\text { (case study \#2) }}$} & $\begin{array}{l}\mathrm{R}=\mathrm{Me} \\
\mathrm{R}=\mathrm{Pr}\end{array}$ & $\begin{array}{l}\mathrm{R}=\mathrm{Et} \rightarrow 01 \\
\mathrm{R}=\mathrm{Bu} \rightarrow 11\end{array}$ \\
\hline P6 & 632.4 & 01101100 & $\mathrm{MeOH}+3 \mathrm{mM} \mathrm{AcNH}_{4}$ & $5 \mu \mathrm{L} \mathrm{min}^{-1}$ \\
\hline
\end{tabular}




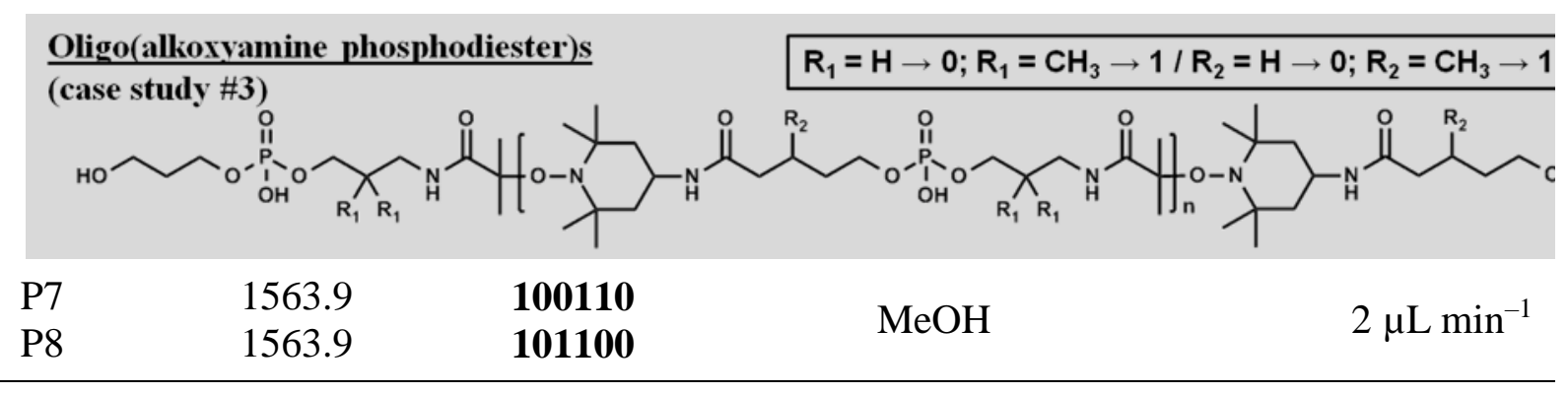

\section{DESI-MS/MS for data storage applications: mapping of coded oligourethanes libraries}

In this first application example, small oligomers were spatially ordered on a DESI plate to write a four-letter word. Because oligomers composing the string of information are not physically mixed, the same species can be used multiple times. This happens to be particularly useful when employing the ASCII code to write binary messages, with each character defined by eight digits. Indeed, each byte is actually composed of two sets of four digits: for lowercase letters, the first four digits are 0110 from a to $\mathrm{p}$ and 0111 from $\mathrm{p}$ to $\mathrm{z}(0100$ from $\mathrm{A}$ to $\mathrm{O}$ and 0101 from $\mathrm{P}$ to $\mathrm{Z}$ for capitalized letters) while the second set includes one of the combinations ranging from 0000 to 1111 (Table S1, Supporting Information). Accordingly, using 4-mers as sub-segments of bytes, a library of twenty oligomers enables coding of all letters of the Roman alphabet as long as those respectively coding for $0100,0101,0110$ and 0111 can be used in a recurrent manner. This is illustrated hereafter, using five different sequence-defined oligourethanes ${ }^{[39]}$ to write the word "styx" (Table 1). The same P1 oligomer (634.4 Da) coding for 0111 (the first four digits of all lowercase letters considered here) was deposited on spots 1, 3, 5 and 7 of a commercial DESI sample plate (Figure S1b, Supporting Information), while oligomers coding for the second set of four digits - P2 with 0011 for "s", P3 with 0100 for " $t$ ", P4 with 1001 for "y", and P5 with 1000 for "x" - were deposited on spots 2, 4, 6 and 8, respectively (top scheme in Figure 1). Of note, P2 and P4 have the same $0_{2} 1_{2}$ co-monomeric composition, and hence the same 620.4 Da mass, while P3 and P5 share the same $0_{3} 1_{1}$ composition at 606.4 Da. 


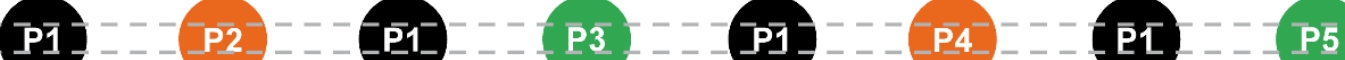

a)

$m / z 605.4$

$m / z 619.4$

$m / z 633.4$

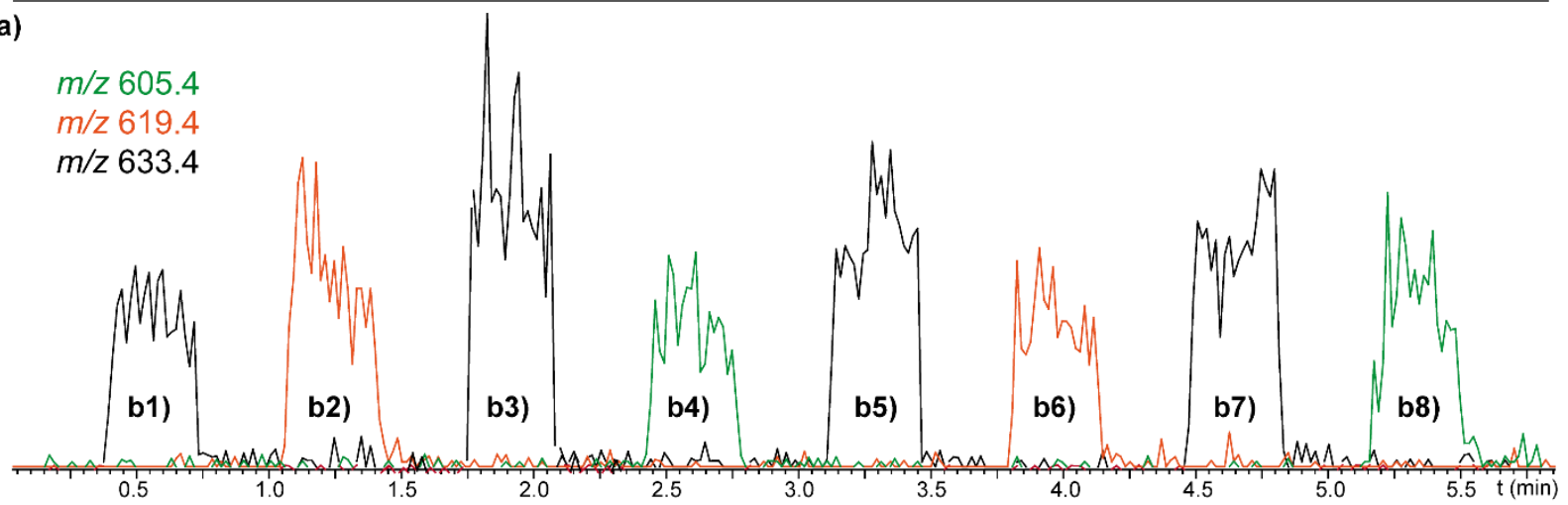

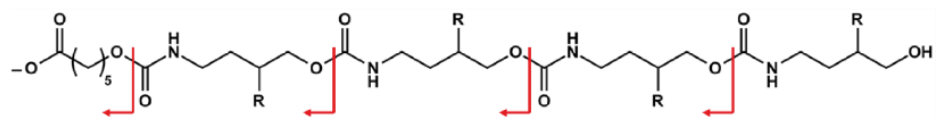

b1)

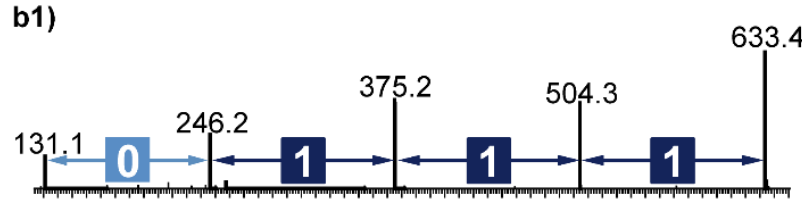

b2)

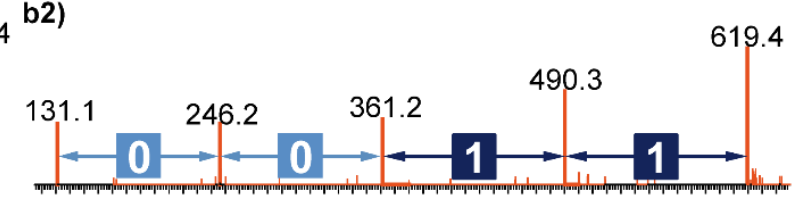

b3)

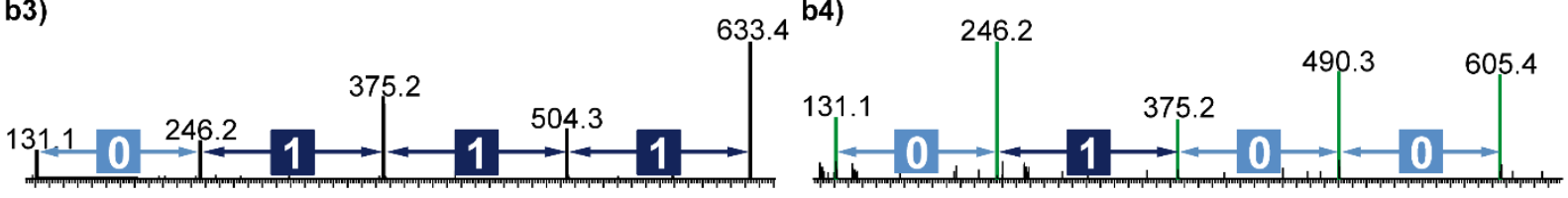

b5)

4 b6)
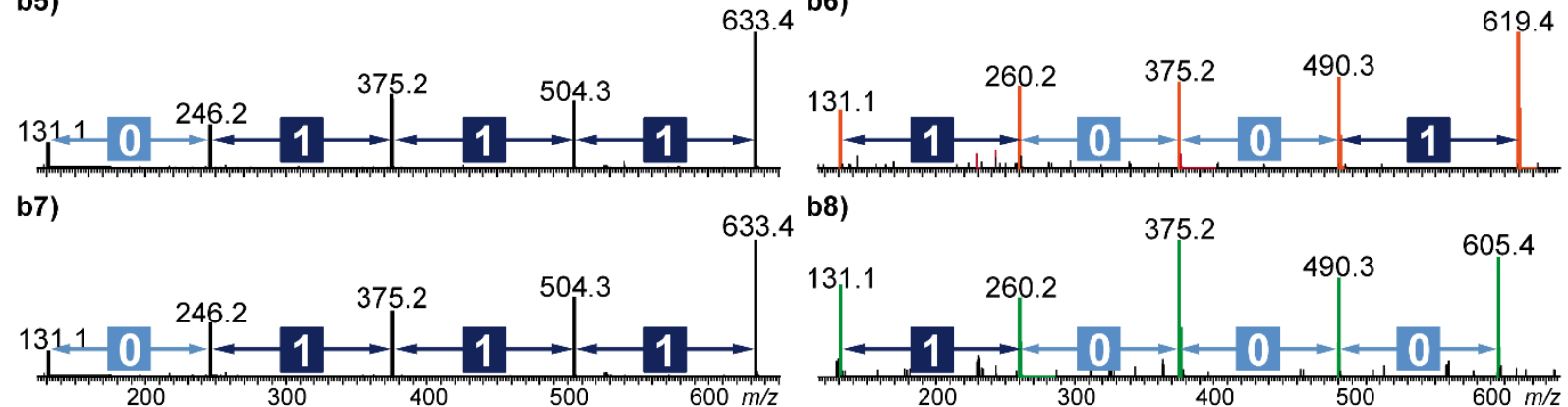

Figure 1. a) Normalized ion chronogram extracted for $\mathrm{m} / \mathrm{z} 605.4$ (in green), $\mathrm{m} / \mathrm{z} 619.4$ (in orange) and $m / z 633.4$ (in black) from the total ion current recorded upon negative mode DESI scanning of the sample plate, which is schematized on top for location of P1 (0111), P2 (0011), P3 (0100), P4 (1001), and P5 (1000). The "bi)" notation of each signal refers to the corresponding MS/MS data shown in b), obtained at a $15 \mathrm{eV}$ collision energy (laboratory frame) according to the dissociation scheme shown on top. Combining pairs of successive binary strings revealed in MS/MS allows the word "styx" to be reconstructed with b1+b2 $\rightarrow 01110011$ $=$ "s", b3+b4 $\rightarrow 01110100=$ "t", b5+b6 $\rightarrow 01111001=$ "y", and b7+b8 $\rightarrow 01111000=$ "x". 
Sample deposition was performed with a $3 \mu \mathrm{L}$ droplet of each working solution (i.e., $~ 1.5$ nmoles of oligomer on each spot) which was air-dried prior analysis. A first time-resolved DESI experiment was performed in the MS mode, electrospraying methanol supplemented with ammonium acetate $(3 \mathrm{mM})$ to promote deprotonation of the $\alpha$ acidic end-group of oligourethanes. Moving the sample plate at a $0.5 \mathrm{~mm} \mathrm{~s}^{-1}$ rate below the DESI spray allows MS data to be recorded from each 3-mm diameter spot during 20 seconds, yielding the total ion current shown in Figure S2a (Supporting Information). Owing to the structure of oligourethanes used here (Table 1), with coding units of mass 115.0 Da for 0 (with $\mathrm{R}=\mathrm{H}$ ) or $129 \mathrm{Da}$ for 1 (with $\mathrm{R}=\mathrm{CH}_{3}$ ), deprotonated $\mathrm{P} 1$ was detected at $\mathrm{m} / \mathrm{z}$ 633.4, $\mathrm{P} 2$ and $\mathrm{P} 4$ isomers at $\mathrm{m} / \mathrm{z}$ 619.4, and P3 and P5 isomers at $m / z 605.4$ (Figure S2b, Supporting Information). This is best evidenced in Figure 1a with the enhanced signal-to-noise ratio achieved in chronograms extracted for each of these anions. All signals exhibit a sharp increase, as a result of instantaneous ionization of oligomers as they are exposed to the DESI spray. Similarly, the sharp signal decrease observed as a given sample spot moves away from the DESI probe clearly indicates that there is neither carryover nor memory effect from one spot to another. Assuming a stable DESI spray, many factors can account for peaks of different intensity in the chronogram of Figure 1a. First, absolute amount of deposited sample may well vary i) since the amount of each working solution $\left(\sim 300 \mathrm{ng} \mu \mathrm{L}^{-1}\right)$ was not strictly controlled and ii) due to systematic $( \pm$ $2.0 \%)$ and random $( \pm 1.3 \%)$ errors associated with the low volume $(3 \mu \mathrm{L})$ of aliquots deposited on the DESI plate. Second, although of the same polymerization degree, oligomers may exhibit slightly different ionization yields as a result of their different co-monomer composition. Increased hydrophobicity is expected for species enriched with units 1 due to their methyl side group: this might favor their release from the secondary droplets leaving the surface, as reported for ESI. ${ }^{[40,41]}$ Third, samples are deposited as droplets that are left air-dried prior to DESI: even when located on the same plate, there is no control on the evaporation rate of the solvent (methanol), which might leave dried residues of different homogeneity levels. This last issue is 
evidenced by hatched pattern at the peak tops instead of the flat plateau expected in case of highly homogeneous deposits.

Nevertheless, ion abundance in MS was sufficiently high for DESI experiments to be performed in the MS/MS mode in order to decipher the binary sequence of each oligomer. As reported earlier, MS/MS sequencing of sequence-coded oligourethanes is remarkably easy in the negative ionization mode, ${ }^{[39]}$ because dissociation pattern of their deprotonated form consists of a series of peaks spaced by the mass of one or the other repeating unit as a result of competitive cleavages of all (CO)-NH bonds (Figure 1b) ${ }^{[42]}$ Hence, starting from the $m / z, 131.1$ product ion which contains the deprotonated $\alpha$ moiety only, the fragment-to-fragment distance corresponds to the mass of either $0(115.0 \mathrm{Da})$ or $1(129.1 \mathrm{Da})$ synthons that are successively found in the sequence. Accordingly, the same 0111 sequence is readily recovered from DESIMS/MS data recorded for P1 on spots 1, 3, 5 and 7 (Figure 1b, left column). MS/MS enables a clear distinction between the 0011 sequence of P2 (Figure 1b2) and the 1001 sequence P4 (Figure 1b6) as well as between P3 (Figure 1b4) and P5 (Figure 1b8) isomers of sequence 0100 and 1000, respectively. Finally, the message is reconstructed by coupling binary strings revealed by MS/MS for two consecutive 4-mers, following the same direction as for DESI scanning. Accordingly, reading the plate from the left- to the right-hand side consists of combining MS/MS outputs from i) spots \#1 (0111) and \#2 (0011) to obtain the 01110011 byte coding for "s", ii) spots \#3 (0111) and \#4 (0100) to have 01110100 for "t", iii) spots \#5 (0111) and \#6 (1001) to obtain 01111001 for “y”, and iv) spots \#7 (0111) and \#8 (1000) to reach 01111000 coding for " $\mathrm{x}$ ".

Although these first results clearly demonstrate the relevance of DESI for $2 \mathrm{D}$ coding approaches (with location as the first dimension and MS/MS as the second dimension), the employed methodology can be further improved. On the one hand, the commercial plate used here offers 3-mm diameter PTFE spots for sample deposition, which is far above the $200 \mu \mathrm{m}$ spatial resolution achieved by DESI on a routine basis. ${ }^{[43]}$ Owing to the high ionization yield of 
these small oligourethanes in the negative mode, their high MS/MS readability and the absence of noticeable spot-to-spot carryover effect, the storage capabilities of DESI plates can easily be increased to enable writing much larger messages than the small "styx" word used here for the sake of simplicity. On the other hand, MS/MS data analysis could be performed on-line using the in-house developed MS-DECODER software, ${ }^{[44]}$ opening perspectives for full automation of the reading process.

\section{DESI-MS/MS for anticounterfeiting applications: tag extraction and decoding}

From their early development, sequence-defined synthetic polymers have rapidly become popular for anticounterfeiting purposes because such applications do not rely on informational macromolecules of high storage capacity but, instead, on small (more readily available) coded species used as tags. However, in analytical workflows reported in this field, the rate-limiting step is undoubtedly tag extraction. Our group used a 10-15 min solvent sonication method to extract small amounts of oligourethane incorporated in commodity polymers, ${ }^{[45]} 3 \mathrm{D}$-printed photo-cured methacrylate resins, ${ }^{[39]}$ or acrylate-based intraocular implants. ${ }^{[46,47]}$ Sonicating pieces of tagged materials in small volumes $(200 \mu \mathrm{L}$ to $3 \mathrm{~mL})$ of solvent supplemented with the appropriate salt permits direct ESI-MS/MS experiments (that is, with no need for additional dilution of the extract). Boukis et al. developed a multiple step protocol of more than 2 hours to recover a molecular tag hidden under the stamp of an envelope and containing the key to decrypt the enclosed message. ${ }^{[18]}$ The stamp area was cut into small pieces and subjected to i) liquid phase extraction $(3 \times 15 \mathrm{~mL}$ of methanol for 15 min followed by $3 \times 15 \mathrm{~mL}$ of dichloromethane for $15 \mathrm{~min}$ ), ii) concentration of the combined extracts under reduced pressure, and iii) purification on a solid phase cartridge prior to ESI-MS/MS decoding of the molecular key. ${ }^{[18]}$ Holloway et al. used molecular pin codes to label bank notes that had to be suspended for 5 hours in ethanol to extract the encrypted tags, prior revealing them via heating in the presence of a trapping agent before ESI-MS. ${ }^{[48]}$ 
In contrast, ambient desorption of coded oligomers from surfaces with DESI droplets can be advantageously employed as an instantaneous extraction step, as previously reported for forensic analysis of inks ${ }^{[49]}$ or detection of anti-theft dye on banknotes. ${ }^{[50]}$ This is demonstrated hereafter with a secret code written in a sequence-defined oligomer deposited in the gummed part of the top flap of an envelope, a safe zone to prevent sample loss during mail transportation and handling. For the sake of validation, two different envelops have been employed. Envelop \#1 was used to record DESI-MS(/MS) data from both blank $\left(\mathrm{g}_{0}\right)$ and tagged $(\mathrm{g})$ areas of the gummed zone before sealing (Figure 2a, left). This sacrificial envelop was also used to evaluate the influence of the gum on DESI efficiency as compared to the paper throat part, either tagged (p) or not $\left(\mathrm{p}_{0}\right)$. Envelop \#2 was tagged in the $\mathrm{g}$ zone only, sealed for a week, then carefully opened to prevent any piece of the seams to remain pasted on the top flap. This second envelop was then exposed to the DESI probe to explore the g' zone of the top flap for subsequent MS/MS decoding of the tag (Figure 2a, right). On the gummed part, the deposited $6 \mu \mathrm{L}$ droplet of the working solution of P6 (i.e, 3 nmoles) was observed to spontaneously spread over a small area of about $1 \mathrm{~cm}^{2}$. For the sake of comparison, the pipette tip was employed to spread the same tag aliquot on a similar area of the paper part. Of note, envelop \#1 was tagged on the day when envelop \#2 was unsealed so that both can be analyzed in the same experimental conditions. 


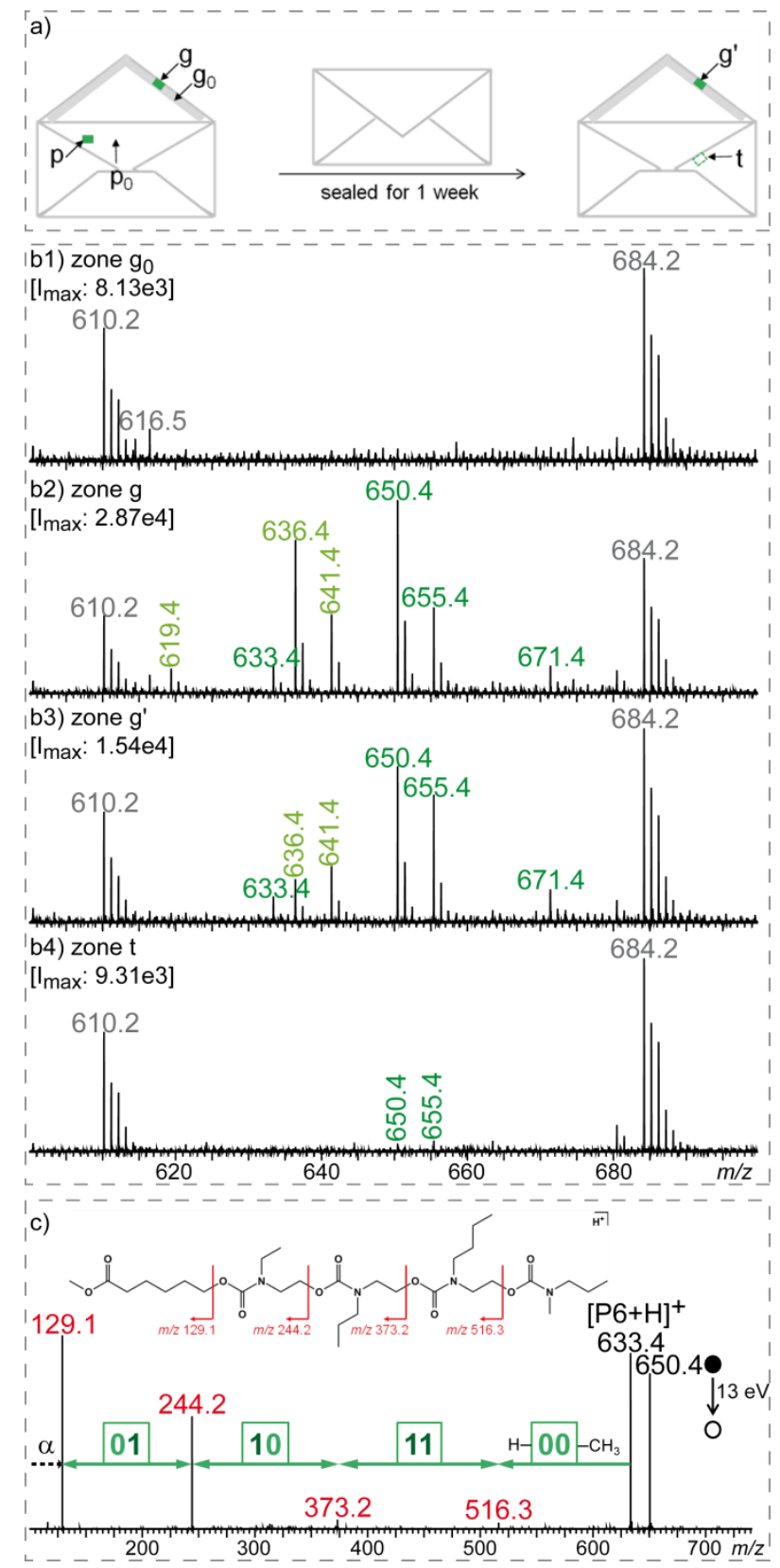

Figure 2. a) Distribution of zones explored by DESI-MS(/MS) on the envelops, on the paper throat part either tagged $(\mathrm{p})$ or not $\left(\mathrm{p}_{0}\right)$, in the gummed area either tagged ( $\mathrm{g}$, before sealing; $\mathrm{g}$, after sealing) or not $\left(\mathrm{g}_{0}\right)$, and in the seam zone to check for transfer $(\mathrm{t})$. Cationic adducts of P6 (632.4 Da) are annotated in dark green while those of its non-methylated precursor (618.4 Da) are in pale green, and chemical noise signals are in grey. Mass spectra recorded during positive mode DESI of b1) zone $g_{0}, b 2$ ) zone $g$, b3) zone g', and b4) zone t, with the major peak intensity reported as $\mathrm{I}_{\max }$ in the top left corner. c) DESI-MS/MS recorded for $\left[\mathrm{P} 6+\mathrm{NH}_{4}\right]^{+}$at $\mathrm{m} / \mathrm{z} 650.4$ in the g' zone after the envelop has been sealed for one week. 
The P6 oligomer selected for this study was an oligo(N-substituted urethane), a recently developed family of sequence-defined macromolecules with the code implemented in the alkyl N-substituents (Table 1). ${ }^{[1]}$ These species are prepared with a set of four monomers, with methyl, ethyl, propyl or butyl side chain defining 00 (101.0 Da), 01 (115.1 Da), 10 (129.1 Da) and $11(143.1 \mathrm{Da})$ binary dyads, respectively. Accordingly, this enhanced alphabet allows n bits of information to be stored in macromolecules with polymerization degree of $n / 2$. In contrast to oligourethanes, oligo(N-substituted urethane)s are best MS/MS sequenced in the positive ion mode after their acidic $\alpha$ end-group has been methylated. ${ }^{[12]}$ DESI-MS data recorded from the gummed zone are reported in Figure 2b. As compared to the blank obtained from zone $\mathrm{g}_{0}$ in envelop \#1 (Figure 2b1), the targeted 632.4 Da species is clearly detected as multiple adducts (all annotated in dark green in Figure 2b2). Formation of $[\mathrm{P} 6+\mathrm{H}]^{+}$at $m / z 633.4$ and $\left[\mathrm{P} 6+\mathrm{NH}_{4}\right]^{+}$at $\mathrm{m} / \mathrm{z} 650.4$ is consistent with the use of ammonium acetate in the methanolic solution sprayed by DESI, whereas alkali that promotes $[\mathrm{P} 6+\mathrm{Na}]^{+}$at $\mathrm{m} / z 655.4$ and $[\mathrm{P} 6+\mathrm{K}]^{+}$at $m / z 671.4$ are either from the solvent or the envelope substrate. Adducts of the $618.4 \mathrm{Da}$ acidic precursor of this ester-terminated P6 are also observed (annotated in pale green in Figure 2b2), revealing incomplete methylation. The same ion series were desorbed from the p paper zone (Figure S3, Supporting Information) although with a higher abundance, with increasing factors ranging from about 5 for $[\mathrm{P} 6+\mathrm{K}]^{+}$to 10 for $\left[\mathrm{P} 6+\mathrm{NH}_{4}\right]^{+}$. Interestingly, the same background ions were detected from $\mathrm{g}_{0}$ and $\mathrm{p}_{0}$ blank areas, as best observed on the full range mass spectra (Figure S4, Supporting Information), but their abundance was about twice higher from the paper zone. This result suggests that, although not ionized by DESI, the gum components affect to some extent (but do not prevent) the oligomer desorption/ionization process. DESI-MS data recorded for the same zone after envelop \#2 has been sealed for one week (g' in Figure 2a) are shown in Figure 2b3. The targeted tag is clearly detected although with less abundance compared to zone $g$ (Figure 2b2): the average decrease is about $41 \%$ but reaches $57 \%$ for $\left[\mathrm{P} 6+\mathrm{NH}_{4}\right]^{+}$at $\mathrm{m} / \mathrm{z}$ 650.4 while it is of $22 \%$ for the $[\mathrm{P} 6+\mathrm{Na}]^{+}$ion at $\mathrm{m} / \mathrm{z} 655.4$. Nevertheless, these ions are produced 
with sufficient abundance to record very good quality MS/MS data and hence reveal the oligomer sequence after one week sealing (Figure 2c). Finally, in order to check for any transfer of the tag on the seams while envelop \#2 was sealed, the zone noted $t$ in Figure 2a was also exposed under the DESI probe. As documented in Figure 3b4, only traces of P6 could be found in this area.

In summary, using DESI, the coded oligomer initially deposited in the gummed part of the envelope was deciphered in about $1 \mathrm{~min}(30 \mathrm{sec}$ for MS $+30 \mathrm{~s}$ for MS/MS) after one week sealing. Owing to ion abundance measured in the MS mode, much higher throughout should certainly be attained. Moreover, DESI enabled an extremely selective surface sampling with no spectral interference observed from the gum components. It should however be acknowledged that a pencil was used to indicate the apexes of each tagged zone so that it can be readily retrieved, particularly after the one week sealing. Yet, blind searching is also possible thanks to the imaging capabilities of the DESI technique, as illustrated hereafter.

\section{DESI-MS/MS for identification applications: imaging of messages written with coded inks}

Shortly after its development, DESI became very popular for imaging analytes distributed on surface samples, and applications range from the earliest MS analysis of dyes on thin layer chromatography plates ${ }^{[51]}$ to the most advanced mapping of molecules in biological tissues. ${ }^{[43]}$ In mass spectrometry imaging (MSI) experiments, small areas (defined as pixels) of the surface are inspected sequentially in time and spectral data recorded for each pixel is stored together with its spatial coordinates. Once the entire surface of interest is scanned, distribution of specific ions can be visualized by the creation of chemical images.

Here, we used two sequence-defined oligomers as inks to draw a small “01” picture. Selected species are dyad-encoded oligo(alkoxyamine phosphodiester)s, ${ }^{[17]}$ with repeating units coding for 2 bits and one bit contained in each chain termination (Scheme S1, Supporting Information). We purposely chose two oligomers (P7 and P8 in Table 1) with the same mass but different 
sequence to draw each digit so that different readouts can be achieved depending on the reading mode (MS vs MS/MS). The design of these sequence-defined oligomers was optimized so that all repeating units can be simultaneously ionized via phosphate deprotonation, a key condition to achieve the simplest MS/MS pattern, ${ }^{[13,52]}$ with exclusive homolysis of alkoxyamine bonds between repeating units leading to a useful ranking of c fragments (Figure S5, Supporting Information). DESI exhibits similar propensity to multiple charging as compared to ESI, so the 3-mers P7 and P8 (1563.9 Da) could readily be generated as triply deprotonated species $(\mathrm{m} / \mathrm{z}$ 525.0) by DESI. However, although a preferential 3- charge state is indeed observed when averaging MS data over 30 seconds (Figure 3a), time-resolved DESI-MS clearly shows that doubly charged oligomers $(\mathrm{m} / \mathrm{z} 787.9)$ were best formed at the early stages (10 first seconds) of the experiment (Figure 3b). These data were not recorded in the imaging mode but by oscillating the DESI probe over the sample. This suggests that deprotonation of phosphate groups was best achieved when increasing the residence time of the oligomers in the thin liquid film formed with the earliest sprayed methanol droplets. Owing to the $300 \mu \mathrm{m} \mathrm{s}^{-1}$ scanning velocity planned to be used for imaging experiments, best sensitivity is expected when selecting ions at the 2- charge state for DESI-MS(/MS) since their abundance is 2.7 times higher than their triply deprotonated homologues after 1 second (Figure 3b). Although slightly less convenient to read than MS/MS data of fully deprotonated species, CID spectra recorded for doubly charged precursors allow sequencing of both species, and the 100110 sequence of P7 (Figure 3c1) to be distinguished from the 101100 sequence of P8 (Figure 3c2) based on product ions $\mathrm{c}_{2}{ }^{\bullet-}$ and $\mathrm{y}_{1}{ }^{\bullet-}$. 

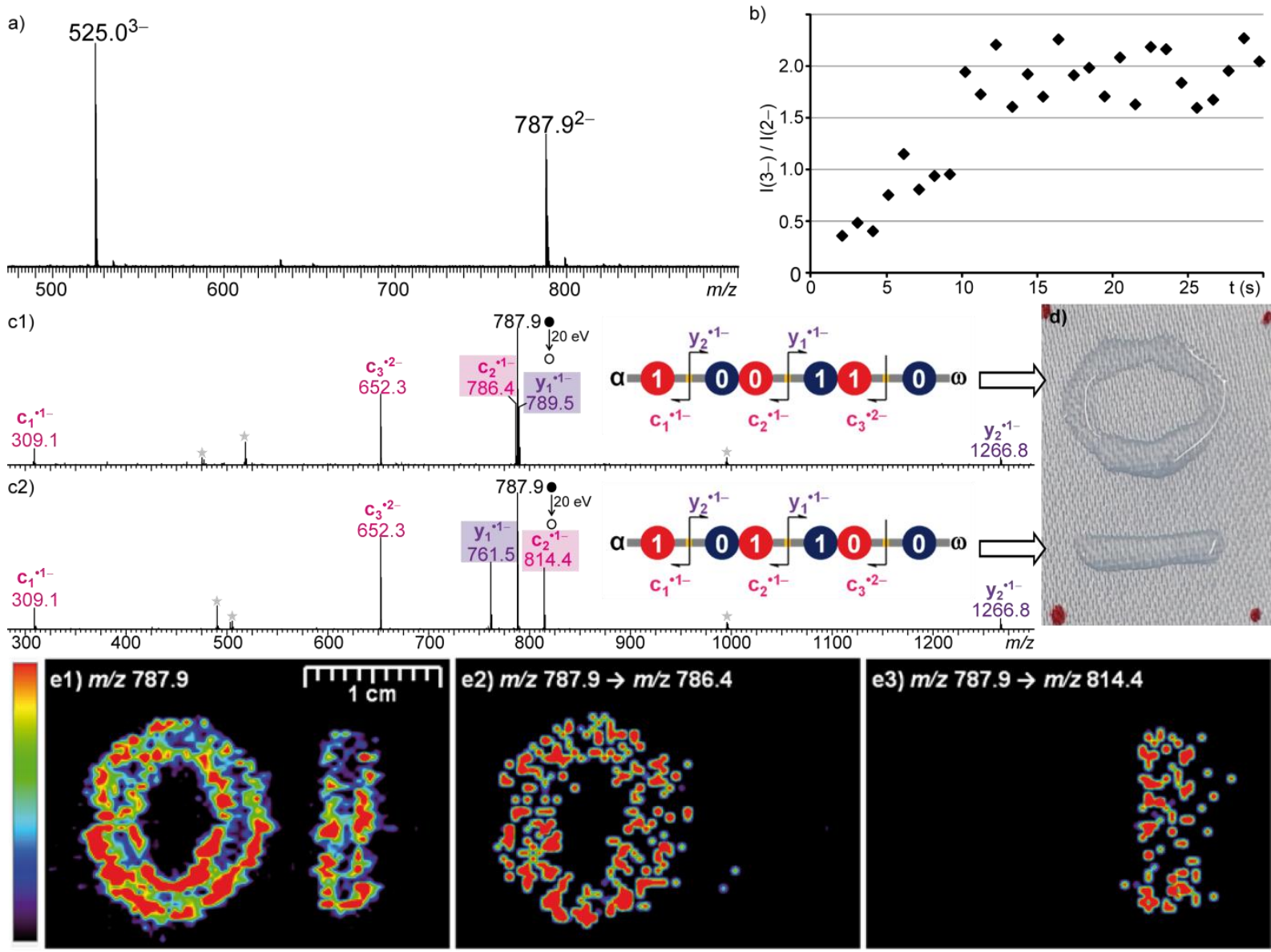

Figure 3. a) Negative mode DESI mass spectrum of P7 averaged over 30 seconds. b) Intensity ratio of $[\mathrm{P} 7-3 \mathrm{H}]^{3-}$ to $[\mathrm{P} 7-2 \mathrm{H}]^{2-}$ measured in DESI-MS as a function of time. DESI-MS/MS of c1) $[\mathrm{P} 7-2 \mathrm{H}]^{2-}$ or $\left.\mathrm{c} 2\right)[\mathrm{P} 8-2 \mathrm{H}]^{2-}$, using a $20 \mathrm{eV}$ collision energy (laboratory frame) to activate the $m / z 787.9$ precursor ion. Inset dissociation schemes show assignments of c (in pink) and y (in purple) product ions in each spectrum, using colored squares to highlight $\mathrm{c}_{2}{ }^{\bullet 1-}$ and $\mathrm{y}_{1}{ }^{\bullet 1-}$ ions that allow the 100110 sequence of $\mathrm{P} 7$ to be distinguished from the 101100 sequence of P8. d) Optical image of the satin fabric after drawing the " 0 " and " 1 " digits with methanolic solution of P7 and P8, respectively. The picture was rotated $90^{\circ}$ clockwise for the sake of presentation. Two-dimensional DESI images of e1) the $\mathrm{m} / \mathrm{z} 787.9$ ion in the MS mode corresponding to both $[\mathrm{P} 7-2 \mathrm{H}]^{2-}$ and $[\mathrm{P} 8-2 \mathrm{H}]^{2-}$ and of the MS/MS reaction $m / z 787.9 \rightarrow \mathrm{c}_{2}{ }^{\bullet-}$ with e2) $\mathrm{c}_{2}{ }^{\bullet 1-}$ at $m / z 786.4$ revealing " 0 " drawn with P7 and e3) $\mathrm{c}_{2}{ }^{\bullet 1-}$ at $\mathrm{m} / \mathrm{z} 814.4$ revealing "1" drawn with P8. 
Employing a pipette tip as a pen, methanolic solutions of each oligomer $\left(\sim 300 \mu \mathrm{g} \mathrm{mL}^{-1}\right)$ were then used to draw "0" (15 $\mu \mathrm{L}$ of P7) and "1" (10 $\mu \mathrm{L}$ of P8) on a piece of satin fabric, (Figure 3d), the two digits being no longer eye-readable after air-drying (Figure S6a, Supporting Information). The actual influence of the substrate on DESI efficiency is still not fully understood, ${ }^{[53]}$ and this satin fabric was empirically found to enable best DESI ion yields for oligo(alkoxyamine phosphodiester)s as compared to other tested materials. The piece of fabric was then fixed on the moving stage with double-sided tape, and DESI imaging was first performed in the MS mode, scanning the $2.3 \mathrm{~cm}$ width x $1.6 \mathrm{~cm}$ height surface at a $300 \mu \mathrm{m} \mathrm{s}^{-1}$ velocity on 52 raws in order to define pixels of about $300 \mu \mathrm{m}$ (Figure S6b, Supporting Information). The image extracted for the targeted $\mathrm{m} / \mathrm{z}, 787.9$ species (the most abundant ion ever detected in these experiments) is documented in Figure 3e1 which shows that the "01" picture is perfectly readable. Based on the associated color scale showing the highest ion abundances in red (left-hand side of Figure 3e1), this chemical image reveals that each oligomer is not homogeneously distributed over the layout of the pipette tip used for deposition of methanolic aliquots, most probably because of drying effects on the fabric. The same experimental conditions were then used to perform DESI imaging in the MS/MS mode, selecting the doubly charged $\mathrm{m} / \mathrm{z}, 787.9$ ion as the dissociating species. Images extracted for specific fragments of $\mathrm{P} 7$ such as $\mathrm{c}_{2}{ }^{\bullet-}$ at $m / z 786.4$ (Figure 3e2) or $\mathrm{y}_{1}{ }^{\bullet 1-}$ at $m / z 789.5$ (Figure S7a, Supporting Information) only show the " 0 " digit drawn with this ink and no trace of the "1" digit. Instead, the "1" digit is revealed in images extracted for $m / z 814.4$ (Figure 3e3) or $m / z 761.5$ (Figure S7b, Supporting Information) respectively corresponding to $\mathrm{c}_{2}{ }^{\bullet 1-}$ and $\mathrm{y}_{1}{ }^{\bullet-}$ fragments diagnostic of P8.

With the $300 \mu \mathrm{m} \mathrm{s}^{-1}$ scanning rate used here, the whole data set requested to build chemical images shown in Figure 3 was recorded in about two hours (62.4 min in each mode). More particularly, only one run was necessary in MS/MS to obtain selective images of the two digital inks because they were isomers. Of course, the " 01 " picture remains a simplistic example 
chosen for the sake of clarity, but it opens vast perspectives for more sophisticated strategies. For example, secret communications can be envisaged based on steganography, where different isomeric inks are selected to drawn pictures or write messages that can only be revealed by monitoring specific MS/MS transitions while remaining unnoticeable in DESI-MS images.

\section{Conclusions}

The three case-studies reported herein show the great potential of DESI as an ionization technique coupled to mass spectrometry for efficient readout of digital polymers organized on surfaces. The first example demonstrates that the low storage capacity of sequence-defined oligomers can advantageously be compensated by bi-dimensional storage, using DESI-MS/MS to reconstruct a complete ASCII-coded word written with small digital chains containing subsegments of bytes and spatially ordered on a surface. Throughput of this approach can further be improved by developing a data acquisition method that enables successive selection of different precursor ions to achieve the same output in the imaging mode, as depicted in the last example. Advances in technologies for spotting should also greatly improve surface storage capacity. DESI also allowed a major step forward to be achieved in anticounterfeiting applications, with selective and ultrafast surface extraction of oligomeric tags exemplified in the second case study. It is also worth mentioning that DESI is non-destructive towards sample substrates, allowing confident and safe analysis of questioned objects or documents.

\section{Experimental Section}

Chemicals. Methanol, acetonitrile and water (all PHLC grade) were purchased from SDS (Peypin, France. Ammonium acetate and formic acid were used as received from Sigma Aldrich (St Louis, MO). Sequence-defined oligomers used in this study (Table 1) were all prepared by solid-phase iterative chemistry. Digital oligourethanes (P1-P5) were synthesized according to 
a previously published protocol, ${ }^{[39]}$ using a simple protecting-group-free iterative approach. ${ }^{[54]}$ Stock solutions of each species were prepared in methanol $\left(\approx 3 \mathrm{mg} \mathrm{mL}^{-1}\right)$ and further diluted $(1 / 10, v / v)$ in methanol prior to deposition on PTFE plots of a DESI plate (Prosolia, Indianapolis, IN). The P6 oligo(N-substituted urethane) was prepared via a solid-phase iterative protocol including the formation of an activated carbonate and its subsequent chemoselective reaction with the secondary amine group of amino alcohol building blocks. ${ }^{[11]}$ Preparation of oligo(alkoxyamine phosphodiester)s P7-P8 was performed using two successive chemoselective steps, phosphoramidite coupling and radical-radical coupling. ${ }^{[5]}$ Stock solutions of each species were prepared at a $\sim 3 \mathrm{mg} \mathrm{mL}^{-1}$ concentration, in methanol for oligourethanes and oligo(N-substituted urethane) or in a water/acetonitrile (50:50, v/v) binary mixture containing $0.1 \%$ formic acid for oligo(alkoxyamine phosphodiester)s. Individual working solutions were prepared by diluting stock solutions $(1 / 10, \mathrm{v} / \mathrm{v})$ in methanol.

Instrumentation. All analyses were performed on a Synapt-G2 (Waters, Manchester, UK) equipped with a 2D DESI ion source (Prosolia, Indianapolis, IN). For all experiments, the DESI source was operated at $35^{\circ} \mathrm{C}$ with a nebulizing gas $\left(\mathrm{N}_{2}\right)$ pressure of 5 bars and with the same geometrical factors: a $3 \mathrm{~mm}$ distance between the capillary tip and the sample surface, a $4 \mathrm{~mm}$ distance between the solvent capillary and the transfer line, a $0.5 \mathrm{~mm}$ between the transfer line and the sample surface, an incident angle to the surface of $55^{\circ}$ and a sampling angle of $5^{\circ}$ (Figure S1a, Supporting Information). In the negative ion mode, experimental source settings were as follows: capillary voltage, $-2.27 \mathrm{kV}$; sampling cone voltage, $-20 \mathrm{~V}$; extraction cone voltage, $-6 \mathrm{~V}$. In the positive ion mode, these settings were as follows: capillary voltage, +3.0 $\mathrm{kV}$; sampling cone voltage, $+20 \mathrm{~V}$; extraction cone voltage, $+6 \mathrm{~V}$. Composition and flow rate of the sprayed solution were optimized for each type of oligomers (Table 1). Samples were deposited on different substrates (see text) including the PTFE plots of commercial DESI plates from Prosolia (Figure S1b, Supporting Information) as droplets of working solutions ( $300 \mu \mathrm{g}$ $\mathrm{mL}^{-1}$ in methanol) and left air-dried prior DESI operation. Mass spectra were recorded every 1 
s using the orthogonal acceleration time-of-flight (oa-TOF) mass analyzer of the instrument. In the MS/MS mode, precursor ions were selected in a quadrupole, activated in a collision cell filled with argon and so-produced fragments were mass analyzed with the oa-TOF. Instrument control, data acquisition and data processing of all experiments were achieved with the MassLynx 4.1 programs provided by Waters. The Firefly (v.2.1.06) data conversion software was used to generate images that were further treated in Biomap (v. 3.8.04).

\section{Supporting Information}

Supporting Information is available from the Wiley Online Library or from the author.

\section{Acknowledgements}

L.C. thanks the Région Provence-Alpes-Côte d'Azur (Research Program 2016, project ENIGMass) for financial support for the acquisition of the Prosolia DESI source, and acknowledges support from Spectropole, the Analytical Facility of Aix-Marseille University, by allowing a special access to the instruments purchased with European Funding (FEDER OBJ2142-3341). The authors thank the French National Research Agency (ANR project digital LbL grant numbers ANR-19-CE06-0020-01 and ANR-19-CE06-0020-02) and the CNRS for financial support. 


\section{References}

[1] J. F. Lutz, M. Ouchi, D. R. Liu, M. Sawamoto, Science 2013, 341, 1238149.

[2] H. Colquhoun, J. F. Lutz, Nat. Chem. 2014, 6, 455.

[3] R. K. Roy, A. Meszynska, C. Laure, L. Charles, C. Verchin, J. F. Lutz, Nat. Commun. 2015, 6, 7237 .

[4] A. Al Ouahabi, J. A. Amalian, L. Charles, J. F. Lutz, Nat. Commun. 2017, 8, 967.

[5] M. Rutten, F. W. Vaandrager, J. Elemans, R. J. M. Nolte, Nat. Rev. Chem. 2018, 2, 365.

[6] S. Martens, A. Landuyt, P. Espeel, B. Devreese, P. Dawyndt, F. Du Prez, Nat. Commun. 2018, 9, 4451.

[7] A. C. Boukis, M. A. R. Meier, Eur. Polym. J. 2018, 104, 32.

[8] Z. H. Huang, Q. N. Shi, J. Guo, F. Y. Meng, Y. J. Zhang, Y. T. Lu, Z. F. Qian, X. P. Li, N. C. Zhou, Z. B. Zhang, X. L. Zhu, Nat. Commun. 2019, 10, 1918.

[9] J. M. Lee, M. B. Koo, S. W. Lee, H. Lee, J. Kwon, Y. H. Shim, S. Y. Kim, K. T. Kim, Nat. Commun. 2020, 11, 56.

[10] A. Al Ouahabi, M. Kotera, L. Charles, J. F. Lutz, ACS Macro Lett. 2015, 4, 1077.

[11] T. Mondal, V. Greff, B. E. Petit, L. Charles, J. F. Lutz, ACS Macro Lett. 2019, 8, 1002.

[12] L. Charles, T. Mondal, V. Greff, M. Razzini, V. Monnier, A. Burel, C. Carapito, J. F. Lutz, Rapid Commun. Mass Spectrom. 2020, 34, e-8815.

[13] J. A. Amalian, A. Al Ouahabi, G. Cavallo, N. F. Konig, S. Poyer, J. F. Lutz, L. Charles, J. Mass Spectrom. 2017, 52, 788.

[14] B. Liu, Q. Shi, L. Hu, Z. Huang, X. Zhu, Z. Zhang, Polym. Chem. 2020, 11, 1702.

[15] J. F. Lutz, Macromolecules 2015, 48, 4759.

[16] S. C. Solleder, D. Zengel, K. S. Wetzel, M. A. R. Meier, Angew. Chem. Int. Ed. 2016, $55,1204$. 
[17] G. Cavallo, S. Poyer, J. A. Amalian, F. Dufour, A. Burel, C. Carapito, L. Charles, J. F. Lutz, Angew. Chem. Int. Ed. 2018, 57, 6266.

[18] A. C. Boukis, K. Reiter, M. Frolich, D. Hofheinz, M. A. R. Meier, Nat. Commun. 2018, 9, 1439.

[19] K. S. Wetzel, M. Frolich, S. C. Solleder, R. Nickisch, P. Treu, M. A. R. Meier, Commun. Chem. 2020, 3, 63.

[20] E. Laurent, J. A. Amalian, M. Parmentier, L. Oswald, A. Al Ouahabi, F. Dufour, K. Launay, J. L. Clement, D. Gigmes, M. A. Delsuc, L. Charles, J. F. Lutz, Macromolecules 2020, 53, 4022.

[21] C. Laure, D. Karamessini, O. Milenkovic, L. Charles, J. F. Lutz, Angew. Chem. Int. Ed. 2016, 55, 10722.

[22] J. Steinkoenig, R. Aksakal, F. Du Prez, Eur. Polym. J. 2019, 120, 109260.

[23] X. C. Chen, W. P. Huang, K. F. Ren, J. Ji, ACS Nano 2018, 12, 8686.

[24] R. Szweda, M. Tschopp, O. Felix, G. Decher, J. F. Lutz, Angew. Chem. Int. Ed. 2018, $57,15817$.

[25] M. Mrksich, ACS Nano 2008, 2, 7.

[26] B. J. Cafferty, A. S. Ten, M. J. Fink, S. Morey, D. J. Preston, M. Mrksich, G. M. Whitesides, ACS Cent. Sci. 2019, 5, 911.

[27] M. Karas, F. Hillenkamp, Anal. Chem. 1988, 60, 2299.

[28] K. Tanaka, H. Waki, Y. Ido, S. Akita, Y. Yoshida, T. Yoshida, T. Matsuo, Rapid Commun. Mass Spectrom. 1988, 2, 151.

[29] E. Kennedy, C. E. Arcadia, J. Geiser, P. M. Weber, C. Rose, B. M. Rubenstein, J. K. Rosenstein, PloS One 2019, 14, e0217364.

[30] C. E. Arcadia, E. Kennedy, J. Geiser, A. Dombroski, K. Oakley, S. L. Chen, L. Sprague, M. Ozmen, J. Sello, P. M. Weber, S. Reda, C. Rose, E. Kim, B. M. Rubenstein, J. K. Rosenstein, Nat. Commun. 2020, 11, 691. 
[31] Z. Takats, J. M. Wiseman, B. Gologan, R. G. Cooks, Science 2004, 306, 471.

[32] A. Venter, M. Nefliu, R. G. Cooks, Trends Anal. Chem. 2008, 27, 284.

[33] M. Nefliu, A. Venter, R. G. Cooks, Chem. Commun. 2006, 8, 888.

[34] A. T. Jackson, J. P. Williams, J. H. Scrivens, Rapid Commun. Mass Spectrom. 2006, $20,2717$.

[35] J. P. Williams, G. R. Hilton, K. Thalassinos, A. T. Jackson, J. H. Scrivens, Rapid Commun. Mass Spectrom. 2007, 21, 1693.

[36] M. Friia, V. Legros, J. Tortajada, W. Buchmann, J. Mass Spectrom. 2012, 47, 1023.

[37] J. M. Wiseman, D. R. Ifa, Q. Y. Song, R. G. Cooks, Angew. Chem. Int. Ed. 2006, 45, 7188

[38] D. R. Ifa, J. M. Wiseman, Q. Y. Song, R. G. Cooks, Int. J. Mass Spectrom. 2007, 259, 8.

[39] U. S. Gunay, B. E. Petit, D. Karamessini, A. Al Ouahabi, J. A. Amalian, C. Chendo, M. Bouquey, D. Gigmes, L. Charles, J. F. Lutz, Chem 2016, 1, 114.

[40] C. G. Enke, Anal. Chem. 1997, 69, 4885.

[41] N. B. Cech, C. G. Enke, Anal. Chem. 2000, 72, 2717.

[42] J. A. Amalian, S. Poyer, B. E. Petit, S. Telitel, V. Monnier, D. Karamessini, D. Gigmes, J. F. Lutz, L. Charles, Int. J. Mass Spectrom. 2017, 421, 271.

[43] C. P. Wu, A. L. Dill, L. S. Eberlin, R. G. Cooks, D. R. Ifa, Mass Spectrom. Rev. 2013, $32,218$.

[44] A. Burel, C. Carapito, J. F. Lutz, L. Charles, Macromolecules 2017, 50, 8290.

[45] D. Karamessini, S. Poyer, L. Charles, J. F. Lutz, Macromol. Rapid Commun. 2017, 38, 1700426.

[46] D. Karamessini, B. E. Petit, M. Bouquey, L. Charles, J. F. Lutz, Adv. Funct. Mater. 2017, 27, 1604595 . 
[47] D. Karamessini, T. Simon-Yarza, S. Poyer, E. Konishcheva, L. Charles, D. Letourneur, J. F. Lutz, Angew. Chem. Int. Ed. 2018, 57, 10574.

[48] J. O. Holloway, F. Van Lysebetten, N. Badi, H. A. Houck, F. E. Du Prez, Adv.Sci. 2020, 7, 1903698.

[49] D. R. Ifa, L. M. Gumaelius, L. S. Eberlin, N. E. Manicke, R. G. Cooks, Analyst 2007, $132,461$.

[50] D. N. Correa, J. J. Zacca, W. F. D. Rocha, R. Borges, W. de Souza, R. Augusti, M. N. Eberlin, P. H. Vendramini, Forensic Sci. Int. 2016, 260, 22.

[51] G. J. Van Berkel, M. J. Ford, M. A. Deibel, Anal. Chem. 2005, 77, 1207.

[52] L. Charles, G. Cavallo, V. Monnier, L. Oswald, R. Szweda, J. F. Lutz, J. Am. Soc. Mass Spectrom. 2017, 28, 1149.

[53] F. Bianchi, M. Mattarozzi, M. Careri, Anal. Bioanal. Chem. 2020, 412, 3967.

[54] T. T. Trinh, C. Laure, J. F. Lutz, Macromol. Chem. Phys. 2015, 216, 1498.

[55] G. Cavallo, A. Al Ouahabi, L. Oswald, L. Charles, J. F. Lutz, J. Am. Chem. Soc. 2016, $138,9417$. 